\title{
Subband Methods for OFDM Equalization
}

\author{
Damian Marelli and Minyue Fu \\ Department of Electrical and Computer Engineering \\ University of Newcastle, N.S.W. 2308 Australia
}

\begin{abstract}
In this paper we propose two equalization methods for orthogonal frequency-division multiplexing (OFDM) modulation. These methods are intended to be alternatives to the known cyclic prefix equalization (CPE) method, and are derived using ideas from subband identification. The first proposed method is called subband equalization (SBE) and the second one is called enhanced cyclic prefix equalization (ECPE). The performance of the different methods are compared in terms of bit error rate (BER) after equalization, convergence rate and computational cost in the presence of noise and fast fading. Both SBE and ECPE outperform the CPE in terms of BER and convergence rate. The SBE does not require the use of $\mathrm{CP}$, and therefore saves channel capacity, but needs more computation than CPE. ECPE requires the use of $\mathrm{CP}$, but its cost is compatible to that of CPE.
\end{abstract}

\section{INTRODUCTION}

Orthogonal frequency-division multiplexing (OFDM) is a multicarrier modulation technique used in broadband wireless systems and local area wireless networks [1]. The signal to be modulated is usually a sequence of QAM or PSK symbols. The basic idea is to split a signal sequence into a large number of sub-channels by a serial-to-parallel conversion, and modulate them by orthogonal subcarriers. This is an effective way for reducing inter-symbol interference (ISI) caused by multipaths. The ISI can be further reduced by the use of the so-called cyclic prefix (CP) [1], which consists in a block of redundant samples at the beginning of each transmitted frame.

Channel equalization is a key component in OFDM transmission. This is used to remove amplitude and phase distortions caused by the channel. OFDM equalization typically employs a combination of $\mathrm{CP}$ with a standard equalization (e.g., linear equalization or decision feedback equalization) [1]. This method will be referred to as cyclic prefix equalization (CPE).

Subband identification is a system identification technique that was recently proposed for applications where the system to be identified can be modelled as a finite-impulse response (FIR) of large tap size [2]. It is shown in [3] that this technique can achieve the same performance as the classical (sometimes called fullband) identification technique, but with a significantly smaller computational cost.

In this paper, we use the subband identification technique to develop two OFDM equalization methods. The performances of these methods are compared in terms of bit error rate (BER) after equalization, convergence rate and computational cost in the presence of noise and fast fading. A fast convergence rate is important since it reduces the channel capacity devoted for the training signal. The first proposed method is called subband equalization (SBE). The SBE method outperforms CPE, in terms of BER and convergence rate. Also, it does not require the use of $\mathrm{CP}$ and therefore has a more efficient use of the channel capacity and more robust against fast fading. However, the computational cost of this method is several times higher than that of CPE. Given this fact we propose an alternative method called enhanced cyclic prefix equalization (ECPE). This method is generalized from CPE but based on the concept of subband identification. It has a performance comparable to that of SBE, but requires less computation. However, it requires the use of $\mathrm{CP}$.

\section{OFDM AND CPE}

As explained in Introduction, the signal $u^{o}(t)$ to be modulated is split into $C$ sub-channels $U^{o}(k)=\left[u^{o}(k C), u^{o}(k C-\right.$ 1), $\left.\cdots, u^{o}(k C-(C-1))\right]^{T}$ (typically $C=512,1024$ or higher) by serial-to-parallel conversion. Then, The modulated signal $u(t)$ is given by

$$
u(t)=\sum_{c=0}^{C-1} u^{o}(k C-c) e^{j 2 \pi \frac{c}{C} t},(k-1) C<t \leq k C
$$

and can be generated by the use of a $C$-point IFFT. Hence, the signal is transmitted in frames of $C$ symbols. Since the frame rate is $1 / C$ th of the symbol rate, inter-symbol interference due to delay spreads is significantly reduced.

The complete OFDM transmission and receiving scheme is represented in Fig. 1, where $g(q)$ is the discrete-time transfer function of the equivalent baseband channel and is assumed to be FIR with tap size $n_{g}$ (typically $n_{g}=50 \sim 100$ ). The signal $v(t)$ is the equivalent baseband noise. The boxes denoted by SP and PS are serial-to-parallel and parallel-toserial converters, respectively. An equalizer (EQ) is used to compensate the distortion introduced by the channel before the received signal is sent to a detector (DET), which is a simple quantizer. Note that, in practice, some error-control code will be used. Therefore, the equalization and decoding will be somehow related, but this is not taken into account in this paper, since we are just interested in evaluating the equalization performance.

\section{A. Cyclic Prefix Equalization (CPE) Method}

In order to equalize the distortion introduced by the channel, the transmission is arranged in packets of $F$ frames. A training signal is transmitted in the fist $I$ frames (training period), in order to tune an adaptive equalizer, that will be used to equalize the remaining $F-I$ frames (transmission period).

The channel involves both slow fading and fast fading. Fast fading causes the channel to vary from frame to frame, but 


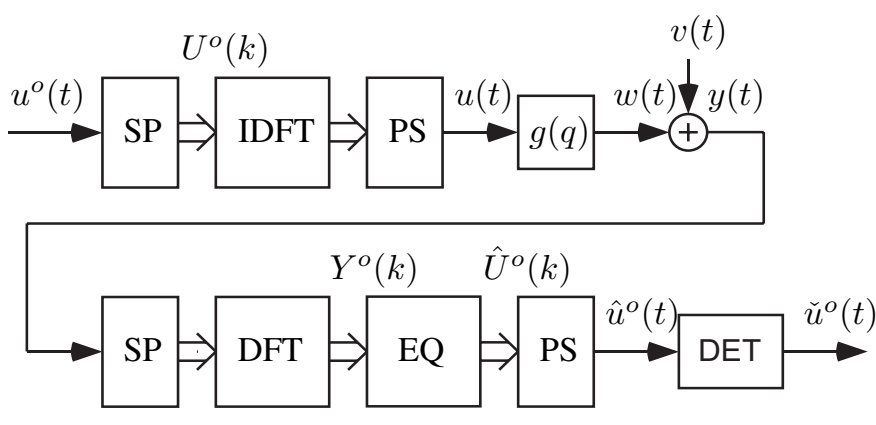

Fig. 1. OFDM Scheme

it mainly affects the ripples in the frequency response of the channel model. Slow fading is the dominant part of the channel distortion, but it can be assumed to change slowly. That is, it can be assumed to be stationary for the duration of the packet. This type of model is regarded as quasi-stationary. For the purpose of equalization, the channel model should capture the slow fading but be insensitive to fast fading. This is the reason for choosing the channel tap size much lower than the frame size.

The so-called cyclic prefix equalization method [4] is commonly used. The idea is to add, at the beginning of each frame of $C$ samples, a block of $P$ samples, which equals the last $P$ samples of the same frame, where $P \geq n_{g}$. It is shown that the mapping from $U^{o}(k)$ to $Y^{o}(k)$ in the absence of channel noise is a constant diagonal matrix. Each of the $C$ sub-channel gains, $\theta_{c}, c=1, \ldots, C$, can be estimated by minimizing the power of the difference $U_{c}^{o}(k)-\theta_{c} Y_{c}^{o}(k)$. Further, equalization is done using these gains directly rather than the FIR channel model.

Since $C \gg n_{g}$, the method above essentially uses overparametrization. Therefore, the computational cost is increased unnecessarily. In practice, only a subset of $n \gtrsim n_{g}$ subchannels are identified, and the other sub-channels are interpolated [1].

The computation of the CPE method involves an FFT, and IFFT, and the recursive least squares (RLS) optimization of the gain coefficient in each of the $n$ sub-channels. Measured in numbers of multiplications, each FFT/IFFT is $\frac{C}{2} \log _{2} C$ per frame, and that of the RLS optimization of one coefficient is 5 per sub-channel. Hence, the overall computational cost of this method, during the training period, is given by

$$
\Psi=\frac{1}{2} \log _{2} C+5 \frac{n}{C} \text { per symbol }
$$

During the transmission (equalization) period, the computational cost becomes

$$
\Psi=\frac{1}{2} \log _{2} C+1 \text { per symbol }
$$

The advantage of the CPE method is two-fold: 1) It is very efficient computationally; 2) When the noise level is low, the equalization error is low (due to the use of $\mathrm{CP}$ ). However, as we will see in the simulations the use of CP makes no significant difference in the final equalization error, in the presence of a high noise level.
The drawbacks of this method are the following: First, a part of the bandwidth (about $5^{\sim} 10 \%$ ) is wasted on CP. Secondly, as we will see that the convergence rate of this method becomes slow in the presence of a moderate to high noise level. Finally, as we will see too that the equalization error is largely affected in the presence of fast fading.

\section{Proposed Methods}

To cope with the drawbacks mentioned above, two methods are proposed. Both of them are in connection with the idea of subband identification.

\section{A. Subband Identification}

The scheme of subband identification is depicted in Fig. 2. The system model $g(q)$ is a discrete-time, time-invariant model, which is assumed to be FIR with tap size $n_{g}$. The idea of subband identification is to split both signals $u(t)$ and $y(t)$ into $M$ subbands using analysis filterbanks $h(q)=\left[h_{1}(q), \ldots, h_{M}(q)\right]^{T}$. These subband signals are down-sampled, by factor of $D$ (i.e. one every $D$ samples is taken), and the results are denoted by two vector signals $U(k)=\left[U_{1}(k), \ldots, U_{M}(k)\right]^{T}$ and $Y(k)=$ $\left[Y_{1}(k), \ldots, Y_{M}(k)\right]^{T}$. The subband parametric model is given by $\hat{G}(q, \theta)=\operatorname{diag}\left\{\hat{G}_{m}\left(q, \theta_{m}\right), \quad m=1, \ldots, M\right\}$, where $\theta=\left[\theta_{1}^{T}, \ldots, \theta_{M}^{T}\right]^{T}$ and $\hat{G}_{m}\left(q, \theta_{m}\right)$ are FIR models of tap size $n_{\hat{G}}$. $\hat{G}(q, \theta)$ is tuned in order to reconstruct $\hat{W}(k, \theta)=$ $\left[\hat{W}_{1}\left(k, \theta_{1}\right), \ldots, \hat{W}_{M}\left(k, \theta_{m}\right)\right]^{T}$. The prediction error $\hat{V}(k, \theta)=$ $\left[\hat{V}_{1}\left(k, \theta_{1}\right), \ldots, \hat{V}_{M}\left(k, \theta_{m}\right)\right]^{T}=Y(k)-\hat{W}(k, \theta)$ is then formed. Finally, an up-sampler (i.e. $D-1$ zeroes inserted between two samples) and a synthesis filterbank $f(q)=\left[f_{1}(q), \ldots, f_{M}(q)\right]^{T}$ are used to reconstruct $\hat{v}(t, \theta)$.

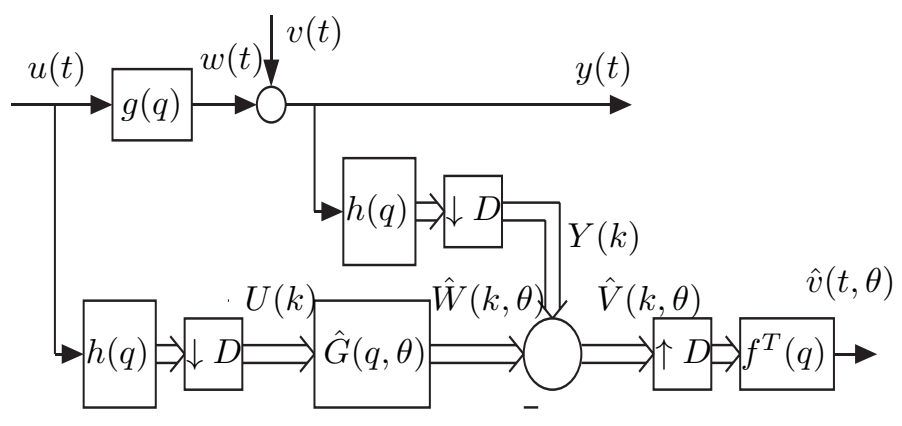

Fig. 2. Subband identification

In [3], it was shown that, with a careful choice of design parameters (number of subbands $(M)$, downsampling factor $(D)$, filterbanks, and subband models), the performance of the subband method, in terms of asymptotic residual error, asymptotic convergence rate and computational cost, can be optimized. We summarize some of these results below:

Let $W(k)=\left[W_{1}(k), \ldots, W_{M}(k)\right]^{T}$ be the downsampled version of $h(q) w(t)$. We define $\tilde{W}(k, \theta)=W(k)-\hat{W}(k, \theta)=$ $\left[\tilde{W}_{1}\left(k, \theta_{1}\right), \ldots, \tilde{W}_{M}\left(k, \theta_{m}\right)\right]^{T}$ and denote by $\tilde{w}(t, \theta)$, the signal obtained by upsampling $\tilde{W}(k, \theta)$ and then filtered using $f(q)$. In the absence of $v(t), \tilde{w}(t, \theta)=\hat{v}(t, \theta)$, which purely 
represents the mismatch between the fullband and subband models. But in general, $\tilde{w}(t, \theta)$ contains a component due to $v(t)$. Define the identification error as the power of the signal $\tilde{w}(t, \theta)$, given by

$$
S_{\tilde{w}}(\theta)=\lim _{T \rightarrow \infty} \frac{1}{T} \sum_{t=1}^{T} \mathcal{E}\left\{|\tilde{w}(t, \theta)|^{2}\right\}
$$

which is a measure of the subband modelling error by $\hat{G}(q, \theta)$. Let $\theta_{N}$ denote the set of parameters computed, by the identification algorithm, up to time $N$. Then, the asymptotic residual error is defined as

$$
S_{\tilde{w}, \lim }=\lim _{N \rightarrow \infty} S_{\tilde{w}}\left(\theta_{N}\right)
$$

In order to identify $g(t)$ with a diagonal subband model, and be able to achieve $S_{\tilde{w}, \text { lim }}=0$, the filterbanks $h(q)$ and $f(q)$ need to take the shapes in Fig. 3. These filters have

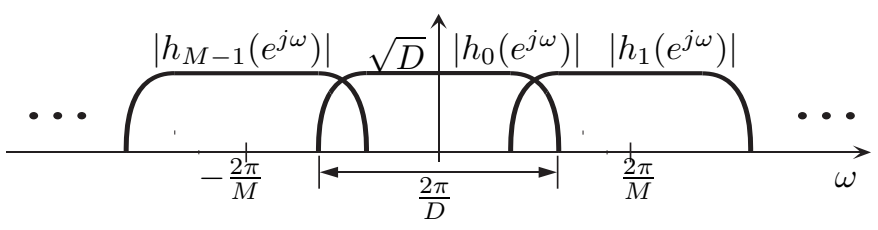

Fig. 3. Ideal filterbank. The shape of the transition bands is proportional to $\sqrt{\omega}$.

zero gain in their stop-band. Therefore, they have an infiniteimpulse response (IIR) and need to be approximated. If we use critical-sampling (i.e. $D=M$ ), the transition bands of the filters become zero. As a consequence, the quality of the approximation will be poor, and therefore $S_{\tilde{w}, \lim }$ will be large. Hence, oversampling $(D<M)$ is used to reduce $S_{\tilde{w} \text {,lim. }}$.

In order to achieve $S_{\tilde{w}, \lim }=0$, not only the filterbanks need to be IIR, but also $n_{\hat{G}}$ needs to be infinite. However, in practice, $S_{\tilde{w} \text {,lim }}$ can be kept reasonably low by choosing

$$
n_{\hat{G}}=\left\lceil\frac{n_{g}}{D}\right\rceil
$$

where $\lceil x\rceil$ denotes the smallest integer larger that $x$.

When $S_{\tilde{w}, \lim }$ is small, the asymptotic convergence rate is given by

$$
\mathcal{E}\left\{S_{\tilde{w}}\left(\theta_{N}\right)-S_{\tilde{w}, \lim }\right\} \lesssim \frac{D n_{\hat{G}}}{N} S_{v}
$$

where $S_{v}$ is the power of the noise signal $v(t)$. The above is valid when $N$ is large and either $n_{\hat{G}}$ is large or $v(t)$ is white.

See [3] for more details on subband identification.

\section{B. Subband Equalization (SBE) Method}

The idea is to apply the subband identification method of Fig. 2, to adapt an equalizer for the communication channel $g(q)$ of the OFDM scheme of Fig. 1. The scheme of the subband method is depicted in Fig. 4. The subband equalizer $\hat{\Gamma}(q, \theta)=\operatorname{diag}\left\{\hat{\Gamma}_{m}\left(q, \theta_{m}\right), m=1, \ldots, M\right\}$ is a diagonal transfer matrix whose entries are FIR filters with tap size $n_{\hat{\Gamma}}$. The blocks AF and SF implement the approximations of the analysis and synthesis filterbanks, respectively.

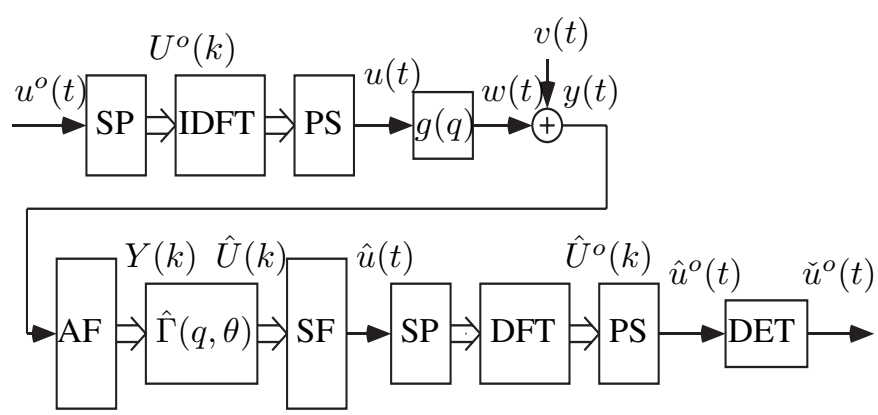

Fig. 4. Subband equalization method

In this case, the signal $\tilde{u}(t, \theta)=u(t)-\hat{u}(t, \theta)$ plays the role of the signal $\tilde{w}(t, \theta)$ in section III-A. We define the asymptotic residual error as

$$
S_{\tilde{u}, \lim }=\lim _{N \rightarrow \infty} \lim _{T \rightarrow \infty} \frac{1}{T} \sum_{t=1}^{T} \mathcal{E}\left\{\left|\tilde{u}\left(t, \theta_{N}\right)\right|^{2}\right\}
$$

and the asymptotic convergence rate as

$$
\mathcal{E}\left\{S_{\tilde{u}}\left(\theta_{N}\right)-S_{\tilde{u}, \lim }\right\}
$$

In the absence of noise, the system to be identified is, $\gamma(q)=g^{-1}(q)$, because this is required for equalization (if $v(t) \neq 0$, the expression of $\gamma(q)$ is different, but a similar reasoning applies). The tap size of the subband equalizer needs to be large enough to model the subband representation of $\gamma(q)$. Although $\gamma(q)$ is IIR in general, it can be approximated by an FIR model. But the tap size $n_{\gamma}$ of this approximation would be much larger that $n_{g}$ in general. As a consequence, in view of (4), $n_{\hat{\Gamma}}$ would be larger that $n_{\hat{G}}$ in general. However, if $D=n_{g}$, then, $n_{\hat{\Gamma}}=n_{\hat{G}}=1$. The reason for this is the following. It is straightforward to prove that, for every subband, the optimal subband equalizer $\hat{\Gamma}_{m}\left(q, \theta_{m}\right)$ is the inverse of the optimal subband model $\hat{G}_{m}\left(q, \theta_{m}\right)$. In view of (4), if $D=n_{g}, \hat{G}_{m}\left(q, \theta_{m}\right)$ can be approximated by a constant, so $\hat{\Gamma}_{m}\left(q, \theta_{m}\right)$ can be approximated by a constant as well. Consequently, we will work with $D=n_{g}$ and $n_{\hat{\Gamma}}=1$. We also note from (5) that further increasing $D$ will worsen the convergence rate. Hence, $D=n_{g}$ is a good choice.

As explained above, CPE will achieve zero asymptotic residual error in the absence of noise, whereas the SBE will not. However, in the presence of a moderate to high noise level (as encountered in practice), both will have similar errors. Further, in the presence of fast fading, the SBE is expected to outperform CPE. This is because CPE will adapt an equalizer by taking into account either all the available $C$ sub-channels or a subset of $n \gtrsim n_{g}$ sub-channels. In the first case, the equalizer captures both slow fading and fast fading. So when the fast fading part changes, equalization errors occur. In the second case, an insufficient number of sub-channels are used to "smooth out" the fast fading effect. In SBE, the choice of $D=n_{g}$ and $n_{\hat{\Gamma}}=1$ yields a subband parametrization of the slow-fading model (i.e. an FIR model of $n_{g}$ taps), but 
this parametrization takes into account all the available subchannels.

We point out that the convergence rate of CPE is much slower than SBE. To understand this, we first note that the presence of $\mathrm{CP}$ does not affect the convergence rate (it only affects the residual error). Secondly, if we remove $\mathrm{CP}$ from the CPE method, then, this method is effectively a criticalsampling subband approach with $M=D=C$ (much larger than the suggested SBE method). In view of (5), the convergence rate of the CPE is slower.

The computational cost of this method is due to the IFFT at the transmitter, the analysis filterbank required to split the signal $y(t)$ into subbands, and the (RLS) optimization of one parameter in $M$ subbands. The subband version of the training signal $u(t)$ does not need to be computed, since it is a known signal. In order to approximate the analysis and synthesis filterbanks, we use a technique called fast-filterbanks. This technique is not detailed in this paper, for space reasons. The only point out that it requires a computational cost of $\log _{2} C+\frac{M}{D}\left(2+\log _{2} \frac{C}{D}\right)$ multiplications per frame. Then, the computational cost during the training period is given by

$$
\Psi=\log _{2} C+\frac{M}{D}\left(7+\log _{2} \frac{C}{D}\right) \text { per symbol }
$$

During the transmission period, the synthesis filterbank and the FFT, shown in Fig. 4, also needs to be computed. Therefore, the computational cost becomes

$$
\Psi=\frac{5}{2} \log _{2} C+\frac{M}{D}\left(5+\log _{2} \frac{C}{D}\right) \text { per symbol }
$$

The SBE method has a few advantages over CPE, as summarized below. First, it does not require the use of $\mathrm{CP}$, which saves bandwidth. Secondly, it has a higher convergence rate. Thirdly, it is more robust in presence of fast fading. These advantages are achieved at a higher computational cost. Further, the concept of subband identification is not as straightforward as the CPE. Next, we introduce another method which is faster and simpler than SBE.

\section{Enhanced Cyclic Prefix Equalization (ECPE) Method}

As explained above, the CPE method can be considered as a critical-sampling subband approach with $M=D=C$, where just $n_{g}$ sub-channels are used to adapt the equalizer. Since just $n_{g}$ parameters have to be identified, in view of (4), it would just make sense to use a down-sampling factor $D$ less than or equal to $n_{g}$. Any further increasing of $D$ would worsen the convergence rate since the same parameters would be adapted less often. To be more precise, the convergence rate for CPE is $K=C / n_{g}$ times slower than that of SBE. In order to speed up the convergence rate, we proceed as follows.

Note that an equalizer can be reconstructed by adapting the parameters of the following set of symbols $\{1, K+$ $\left.1,2 K+1, \cdots,\left(n_{g}-1\right) K+1\right\}$. But, other equalizers can be reconstructed by using the sets of symbols $\{k, K+k, 2 K+$ $\left.k, \cdots,\left(n_{g}-1\right) K+k\right\}, k=2, \cdots, K$. So there are $K$ sets of symbols that can be used for adaptation. The idea of the proposed method is to work with some underlying model of $n_{g}$ parameters, and to adapt these parameters using all the $K$ sets of symbols. This would increase the convergence rate by a factor of $K$, and therefore compensate the decrease introduced by the excessive down-sampling.

To get this underlying parametric model, we proceed as follows. In $\mathrm{CPE}$ we adapt one parameter per sub-channel (i.e. $n=C$ parameters). Then, we apply a $C$-point IFFT to take this frequency domain parameters into its time domain representation. In the time domain we take the first $n_{g}$ parameters, and pad the rest $C-n_{g}$ parameters with zeros. Finally, we apply a $C$-point FFT to this zero-padded time domain sequence to get the final frequency domain parameters. This procedure is equivalent to find the linear minimum mean square estimator of the first $n_{g}$ parameters of the time domain representation of the adaptive equalizer, at each iteration. The same estimation approach has been used in [5], to find a lowrank approxaimation of an optimal (non-adaptive) equalizer, for an OFDM modulation system.

Not only the convergence rate of this method is expected to be faster than that of CPE, but also it is expected to be more robust in presence of fast fading since the parameters are optimized using all the information available. The only drawbacks, with respect to SBE are that it makes use of $\mathrm{CP}$, and therefore the extra bandwidth that it requires. Further, SBE offers some extra robustness. This is due to the overparametrization generated by the use of oversampling.

The computational cost of this method is that of $\mathrm{CPE}$ with $n=C$ because the passage between time domain and frequency domain does not need to be done on every iteration, and can be done at the end of the training period.

\section{Simulation}

In order to compare the performance of the three methods, we equalize an OFDM channel with 4-PSK symbols, $C=$ 1024 and $n_{g}=64$. The spectrum of the channel is shown in Fig. 5

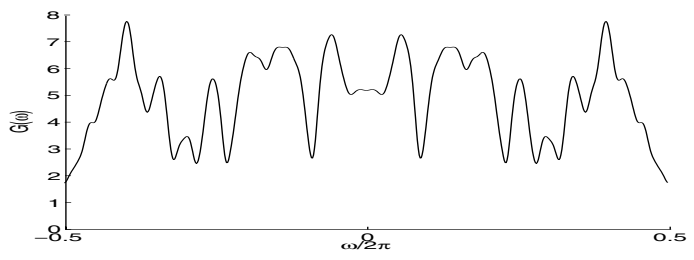

Fig. 5 .

We use two variants of CPE, i.e. with $n=C$ and $n=n_{g}$. For $\mathrm{SBE}$ we use $D=n_{g}$ and $M=2 D$. We compare the performances for different noise levels, with or without $\mathrm{CP}$, and with or without fast fading.

In the first simulation we assume a noise-to-signal ratio of 0.02. In Fig. 6 we use CP while in Fig. 7 it is removed. We can see that, if the $\mathrm{CP}$ is not used, SBE outperforms the other methods. This shows that SBE is the best choice without CP. 


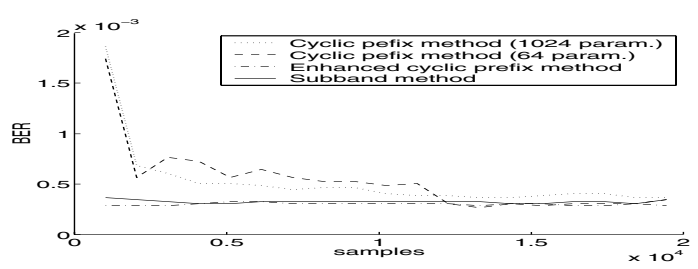

Fig. 6.

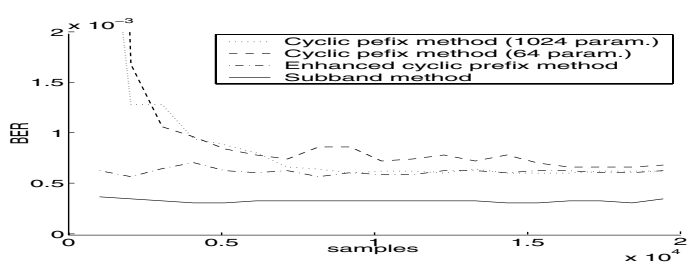

Fig. 7.

In the second simulation, we increase the noise-to-signal ratio to 0.05 . The evolutions of the BER for the four methods, with and without CP, are shown in Figs. 8 and 9, respectively. Fig. 8 shows that, while the final BER is similar for the four

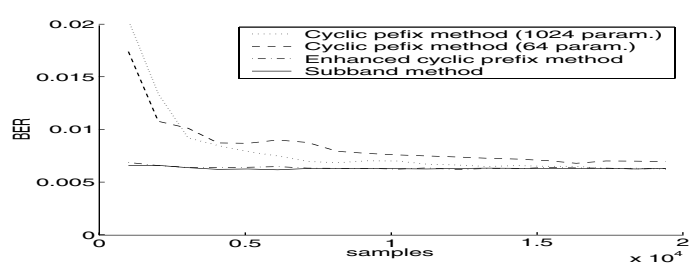

Fig. 8 .

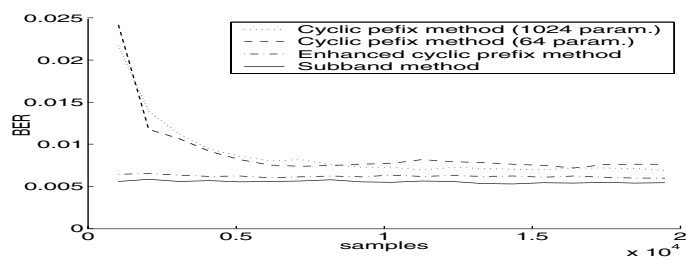

Fig. 9.

methods, the convergence rates of SBE and ECPE are much faster than that of CPE.

In the final simulation, we evaluate the performance in the presence of fast fading. The noise-to-signal ratio is still 0.05 but we have used two fast fading models, one for training and the other for data transmission. The fast fading is simulated by adding a tail to the slow-fading model and its energy is $20 \%$ of the slow-fading part. The model in the training period is shown in Fig 10. The model for the transmission period is similar to Fig. 10. The evolutions of the BER, with and without CP, are shown in Figs. 11 and 12, respectively. It can be seen that the two proposed methods have a more robust behavior.

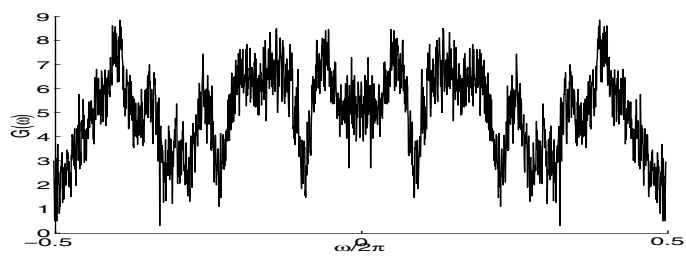

Fig. 10.

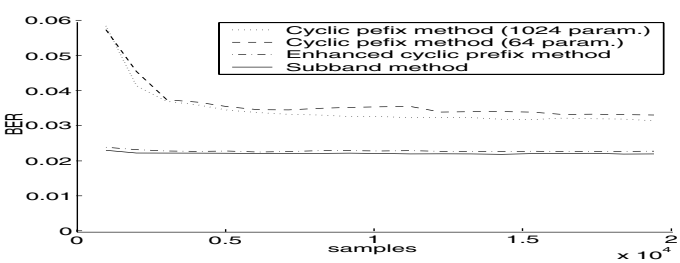

Fig. 11.

The computational costs in the training period are: CPE $(n=64)=5.3, \operatorname{CPE}(n=1024)=10, \mathrm{SBE}=27, \mathrm{ECPE}=10$. For the transmission period: CPE $(n=64)=6, \operatorname{CPE}(n=1024)=6$, $\mathrm{SBE}=54, \mathrm{ECPE}=6$.

\section{Conclusion}

In this paper we proposed two equalization methods for the OFDM modulation scheme. Both outperform the classical $\mathrm{CPE}$, in terms of convergence rate (which implies a smaller number of frames in the training period, and therefore, a more efficient use of the channel capacity) and BER at the presence of noise and fast fading. The simulation results support this fact. The two proposed methods are valid options, since both provide compatible performances, but SBE does not requires the use of $\mathrm{CP}$, while ECPE is computationally more efficient.

\section{REFERENCES}

[1] Richard van Nee and Ramjee Prasad, OFDM wireless multimedia communications, Boston: Artech House, 2000.

[2] Youhong Lu and Joel Morris, "Gabor expansion for adaptive echo cancellation,” IEEE Signal Processing Magazine, pp. 68-80, March 1999.

[3] Damian Marelli and Minyue Fu, "Performance analysis for subband identification," Submitted to IEEE Transactions on Signal Processing.

[4] Zhengdao Wang and Georgios B. Giannakis, "Wireless multicarrier communications," IEEE Signal Processing Magazine, pp. 29-48, May 2000.

[5] Ove Edfos, Magnus Sandell, Jan-Jaap van de Beek, Sarah Kate Wilson, and Per Ola Brjesson, "Ofdm channel estimation by singular value decomposition," IEEE Transactions on Communications, pp. 931-939, July 1998.

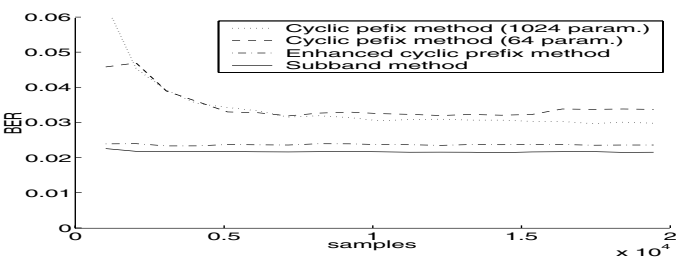

Fig. 12. 